\section{Influência da escolaridade \\ e do trabalho maternos no \\ aleitamento materno exclusivo}

\section{Influence of mothers' schooling and work on the practice of exclusive breastfeeding}

\section{Jorginete de Jesus Damião}

Instituto de Nutrição Annes Dias / Secretaria Municipal de Saúde do Rio de Janeiro. Instituto de Nutrição da Universidade do Estado do Rio de Janeiro INU/UERJ

\section{Resumo}

O presente artigo tem por objetivo analisar a associação entre a prática do aleitamento materno exclusivo (AME) e características maternas (idade, escolaridade, paridade e trabalho), peso ao nascer e alimentação no primeiro dia após alta da maternidade, em crianças menores de quatro meses de idade. Para tal, foram utilizados dados de dois inquéritos realizados no município do Rio de Janeiro, em 1998 e 2000. Nesses inquéritos foram realizadas entrevistas com acompanhantes de menores de um ano de idade, selecionados por meio de amostragem probabilística da população de menores de um ano vacinada nos Dias Nacionais de Vacinação (que possuem cobertura universal para esta faixa etária neste município) ( $\mathrm{n}=2.459)$. A prática do AME foi descrita segundo cada uma das variáveis de exposição e, em seguida, procedeu-se a análise multivariada por regressão logística. A prevalência de AME foi de $22,7 \%$ entre os menores de quatro meses, variando de $39,6 \%$ no primeiro dia a $12,4 \%$ aos 120 dias de vida. Apresentaram maiores prevalências de AME os filhos de mulheres de maior escolaridade, filhos de mulheres que não trabalhavam fora e crianças que estavam em AME no primeiro dia em casa depois da alta da maternidade. Os resultados da análise multivariada indicam associação positiva de AME com escolaridade materna (OR: 1,93 para $3^{\circ}$ grau completo) e negativa com trabalho materno (OR: 0,59). A escolaridade e trabalho maternos e alimentação da criança no primeiro dia em casa após alta da maternidade tiveram associação com o AME entre menores de quatro meses de idade.

Palavras-chaves: Aleitamento materno. Estudos Transversais. Saúde da Criança.

Agradecimentos: A autora agradece ao Dr. Ricardo Ventura Santos e à Dra Inês Rugani Ribeiro de Castro que orientaram a dissertação de mestrado que deu origem a este artigo: Amamentação em crianças menores de um ano no município do Rio de Janeiro: fatores associados à amamentação exclusiva e comparação entre métodos de avaliação da ingestão alimentar [dissertação de mestrado]. Rio de Janeiro: ENSP/ Fiocruz; 2002.

Correspondência: Jorginete de Jesus Damião. Avenida Pasteur, 44 - Botafogo, Rio de Janeiro, RJ CEP 22290-240. E-mail: jdamiao@rio.rj.gov.br 


\section{Abstract}

This article aims at examining the association between the practice of exclusive breastfeeding (EBF) and characteristics of mothers (age, schooling, parity, and work), birth weight, and infant feeding practices on the first day after discharge from the maternity ward in children under four months of age. Data used to this end were collected from two surveys done in the city of Rio de Janeiro in 1998 and 2000. These two surveys involved interviewing mothers with children under one year of age, sampled statistically based on the population of children in that age group vaccinated on national vaccination days (which have universal coverage in this age group in that municipality) $(n=2,459)$. The practice of EBF was described as a function of each of the aforementioned variables, and then multivariate logistic regression analysis was applied. The prevalence of EBF was $22.7 \%$ among children under four months, ranging from $39.6 \%$ on the first day to $12.4 \%$ at 120 days of age. Higher proportions of EBF were found among children whose mothers had a higher level of schooling or who did not work, and children who were exclusively breastfed on the first day at home after discharge from the maternity ward. The results of the multivariate analysis indicate a positive association of EBF with mother's schooling (OR 1.93 for university graduates), and negative with mother's employment (OR 0.59). The results show that this type of monitoring is useful for evaluating and planning actions for the promotion of breastfeeding in the city. Mother's schooling and work, as well as infant feeding practices on the first day after discharge from the maternity ward were shown to be associated with exclusive breastfeeding rates in the first four months of life.

Keywords: Breast-feeding. Cross-sectional studies. Child Health (Public Health).

\section{Introdução}

A manutenção de práticas alimentares adequadas na infância é essencial para a garantia da sobrevida e saúde das crianças, incluindo condições ótimas de estado nutricional, crescimento e desenvolvimento, sendo o aleitamento materno uma das mais importantes práticas promotoras de saúde infantil, tendo reflexos positivos durante toda a vida ${ }^{1}$.

Apesar da intensa mobilização mundial em prol da amamentação, o desmame precoce e a introdução inadequada da alimentação complementar ainda são práticas freqüentes em todo o mundo. No Brasil, não obstante a baixa prevalência do aleitamento materno exclusivo, tem sido observada melhoria nos indicadores da prática de amamentação $0^{2-10}$.

Venâncio e Monteiro ${ }^{6}$ descreveram a trajetória da prática da amamentação no Brasil utilizando os dados de dois inquéritos nacionais, o Estudo Nacional de Despesa Familiar (ENDEF) - 1974/1975 e a Pesquisa Nacional sobre Saúde e Nutrição (PNSN) - 1989. Os autores observaram o aumento da freqüência e duração da amamentação entre os menores de um ano, em todas as idades. A mediana da amamentação no país passou de 2,5 meses em 1975, para 5,5 meses em 1989. Analisando-se as tendências da amamentação segundo área (urbana e rural), região do país, renda e escolaridade materna, observa-se que os incrementos ocorreram em todos os estratos da população, havendo melhores resultados para os grupos que iniciaram o período com menores freqüências de amamentação, quais sejam, crianças da área urbana do país, nas regiões Centro-Oeste e Sudeste, entre as crianças de maior renda e entre as crianças filhas de mães de maior escolaridade. Em conseqüência, houve mudanças nos diferenciais de freqüências de amamentação segundo renda e escolaridade. Em 1975, a duração mediana da amamentação era inversamente relacionada à renda $\mathrm{e}$ à escolaridade, relação que se modificou em 1989.

A pesquisa sobre prevalência do aleita- 
mento materno nas capitais brasileiras e no Distrito Federal realizada em 1999 mostrou, mais uma vez, uma grande heterogeneidade em relação a esta prática no país. A mediana de aleitamento exclusivo foi de 34 dias, variando de 8,3 dias em Cuiabá a 77,3 dias em Fortaleza ${ }^{11}$. Outros estudos de base populacional confirmam estas tendências de melhoria da prática de amamentação e discutem seus diferenciais biológicos e socioeconômicos ${ }^{12-14}$.

Historicamente, a prática da amamentação encontra-se condicionada a diferentes fatores, tais como comportamentos sociais, interesses comerciais e conhecimentos científicos ${ }^{15}$.

No Brasil, estudos recentes têm demonstrado a influência de fatores como escolaridade e idade maternas e práticas associadas ao parto e nascimento sobre a incidência e duração do aleitamento materno ${ }^{13,14,16-19}$. Contudo, fica clara a necessidade de se conhecer os fatores associados ao aleitamento materno em cada realidade, com vistas a subsidiar a escolha de estratégias mais efetivas de promoção da amamentação, que focalizem e adeqüem as intervenções aos grupos de maior risco.

Este artigo tem por objetivo avaliar a associação entre características maternas, peso ao nascer e alimentação no primeiro dia após alta da maternidade e a prática do aleitamento materno exclusivo (AME) em crianças menores de quatro meses de idade no Município do Rio de Janeiro. Ressalta-se que, embora a recomendação da Organização Mundial de Saúde, adotada pelo Ministério da Saúde, seja de que crianças até os seis meses de idade recebam AME, optou-se por estudar os menores de quatro meses de idade, uma vez que sua prática após essa idade ainda é menos freqüente ${ }^{20}$.

\section{Material e Método}

Os dados analisados neste estudo são advindos de dois inquéritos conduzidos em 1998 e 2000, nos quais foram investigadas as práticas alimentares das crianças menores de um ano de idade no município do Rio de
Janeiro $^{21,22}$. Nos dois anos, foram estudadas amostras probabilísticas da população de menores de um ano vacinada durante os Dias Nacionais de Vacinação (que possuem cobertura universal para esta faixa etária neste município), aplicando-se o processo de amostragem por conglomerado auto ponderada, cuja unidade amostral era o posto de vacinação. Para a estimativa do número de crianças a serem entrevistadas em cada conglomerado, utilizou-se como base o número de crianças vacinadas no ano anterior em cada posto de vacinação. Em seguida, realizou-se um processo de amostragem sistemática simples, sendo definido para cada posto de vacinação o intervalo para a amostragem sistemática ${ }^{23}$. Dos cerca de 800 PV's existentes em cada Dia de Vacinação, foram sorteados 74 e 86, em 1998 e 2000, respectivamente, distribuídos por todo o território da cidade.

Dentre as crianças estudadas nos dois inquéritos, 1.203 eram menores de quatro meses em 1998 e 1.271 em 2000. Destas, foram obtidos dados sobre a amamentação para 1.191 e 1.268 crianças, respectivamente, a partir de informações referentes à alimentação atual da criança ("current status").

O desenho desses inquéritos foi delineado com a assessoria do Núcleo de Estudos em Nutrição e Saúde da Universidade de São Paulo (NUPENS/USP) e a amostragem foi definida pelo Serviço de Estatística da Escola Nacional de Saúde Pública da Fundação Oswaldo Cruz (ENSP/FIOCRUZ).

As entrevistas foram realizadas por cerca de 200 voluntários treinados a cada ano, em sua maioria acadêmicos do curso de nutrição, supervisionados por profissionais da Secretaria Municipal de Saúde do Rio de Janeiro e por professores de universidades parceiras. Para chamada dos voluntários houve divulgação do estudo em cursos de graduação da área de saúde em universidades públicas e privadas da cidade. Os voluntários selecionados foram aqueles que participaram do treinamento para coleta de dados e que ao final deste se mostraram aptos para a tarefa.

As entrevistas foram realizadas nos pos- 
tos de vacinação (PV's) da cidade, antes ou depois de a criança ser vacinada, dependendo da dinâmica de funcionamento do PV. Os acompanhantes das crianças selecionadas por meio da amostragem já descrita responderam a um questionário com questões fechadas contendo informações sobre a alimentação atual e no primeiro dia em casa após alta da maternidade, dados sobre o nascimento e características maternas. Os questionários utilizados nos dois anos eram similares, podendo ser comparáveis.

As categorias de aleitamento materno utilizadas neste estudo foram ${ }^{24}$ :

- Aleitamento materno exclusivo (AME) - crianças que recebem somente leite materno, sem água/chá/suco, outro leite ou outros alimentos;

- Aleitamento materno predominante (AMP) - crianças que recebem somente leite materno, com água/chá/suco, sem outro leite ou outros alimentos;

- AMEP - crianças que estão em AME ou AMP;

- Aleitamento materno (AM) - crianças que recebem leite materno, com ou sem complementos.

A análise dos dados foi realizada pela descrição da prática do AME segundo cada uma das variáveis de exposição. Para esta análise foi utilizado o teste de qui-quadrado, considerando um nível de significância de $5 \%$. Em seguida, a medida de associação entre AME e as características maternas e de nascimento e a alimentação no primeiro dia em casa após alta da maternidade foi expressa em odds ratio (OR) e seus intervalos de confiança (IC 95\%), obtidos através de análise multivariada por regressão logística $^{25,26}$, tendo como variável dependente o AME e variáveis independentes: ano de realização do inquérito; peso ao nascer (variável coletada apenas no ano 2000); escolaridade da mãe ( $1^{\circ}$ grau incompleto, $1^{\circ}$ grau completo, $2^{\circ}$ grau completo, $3^{\circ}$ grau completo); idade da mãe - foi estudada na análise univariada segundo faixas etárias ( $<19,20$ a 24,25 a 29, 30 a 34, $>35)$; e, na multivariada, como variável contínua, paridade (primiparidade ou multiparidade); trabalho materno - a mãe estar ou não exercendo atividade remunerada que fizesse com que se afastasse da criança pelo menos um dia por semana; categorias de aleitamento no primeiro dia em casa - estar ou não em AME, AMP ou AM no primeiro dia após alta da maternidade (análise restrita às crianças menores de quatro meses e que tiveram alta da maternidade com até quatro dias após o nascimento); IHAC - local de nascimento credenciado à Iniciativa Hospital Amigo da Criança.

A partir do modelo de regressão logística, estimou-se a probabilidade de as crianças estarem em AME no primeiro dia de vida e aos 30, 60, 90 e 120 dias de vida.

Para a análise univariada foi utilizado o software Epi-info versão 6.04 e, para a análise multivariada, o software Stata $6.0^{27,28}$.

\section{Resultados}

A distribuição de idade materna nos nascidos vivos residentes no município do Rio de Janeiro em 1998 e 2000 (obtidas através do Sistema de Informação sobre Nascidos Vivos - SINASC) é comparável à distribuição desta característica nas crianças que fizeram parte da "Pesquisa sobre práticas alimentares no primeiro ano de vida" nos dois anos (1998 e 2000), assim como a distribuição de peso ao nascer, variável coletada somente no segundo ano de estudo, em 2000. Isto autoriza considerar que os resultados obtidos nas amostras estudadas representam de fato a população carioca menor de um ano.

A variável ano de realização do inquérito não alterou a probabilidade de as crianças menores de quatro meses estarem em AME $(\mathrm{OR}=1,031 ; \mathrm{IC}=0,824-1,291 ; \mathrm{p}=0,787)$, sendo, portanto, possível a junção dos bancos de dados de 1998 e 2000 para as análises pretendidas. Portanto, as análises geradas referem-se a um banco de 2.459 crianças menores de quatro meses, com exceção da variável peso ao nascer, coletada apenas em 2000, para a qual se obteve informação para 1.265 crianças. A freqüência de aleitamento materno exclusivo, no grupo estudado, foi 
de 22,7\%. A partir do modelo de regressão logística estimou-se a probabilidade das crianças estarem em AME em idades específicas. A probabilidade de AME variou de $39,6 \%$ no primeiro dia a $12,4 \%$ aos 120 dias vida (Tabela 1).

A distribuição das crianças segundo características maternas e relacionadas ao nascimento das crianças está apresentada na Tabela 2.

Analisando-se as informações sobre as práticas alimentares das crianças no primeiro dia após alta da maternidade, observou-se que, no seu primeiro dia em casa, a grande maioria $(96,3 \%)$ mamava no peito. Sendo que, destas, $67,0 \%$ estavam em AME, 18,6\% em AMP e 14,4\% já ingeriam outro tipo de leite ou fórmula infantil.

A análise univariada da associação entre AME e características maternas e do nascimento pode ser observada na Tabela 3 e entre AME e a alimentação no primeiro dia após alta da maternidade na Tabela 4. Ressalta-se que na Tabela 4 as crianças foram agrupadas segundo a alimentação no primeiro dia em casa após alta da maternidade, sendo apresentadas as freqüências de AME no momento do inquérito para cada grupo.

A prática do aleitamento materno exclusivo mostrou-se associada à escolaridade e trabalho maternos. Em relação à primeira característica, as mães de maior escolaridade tiveram maiores freqüências de AME ( $p=0,001)$. Em relação ao trabalho materno, entre os filhos de mulheres que não trabalhavam, a freqüência de AME era o dobro daqueles cujas mães, no momento da entrevista, tinham alguma atividade ocupacional que as faziam ficar afastadas de casa $(p=0,000)$. Ainda que tenham sido observadas tendências de maior prevalência de AME com aumento da idade materna e entre crianças que nasceram em Hospitais Amigos da Criança, essas diferenças não foram significativas (Tabela 3).

Ter recebido amamentação exclusiva no primeiro dia em casa após alta da maternidade teve associação com AME no momento do inquérito (current status). O grupo de crianças que estavam em AME no primeiro dia em casa, apresentou freqüência quase três vezes superior desta prática no momento do inquérito, quando comparado ao grupo que havia recebido algum alimento, em substituição ou em complementação ao leite materno no primeiro dia em casa (Tabela 4).

Como na análise univariada possíveis fatores de confusão não estão controlados, efetuou-se análise de regressão logística multivariada para identificar os fatores de risco independentes para o AME, cujos resultados estão mostrados na Tabela 5.

Como já foi descrito, a continuidade do AME esteve associada com a prática de AME no primeiro dia em casa, que, por sua vez, teve associação com a escolaridade

Tabela 1 - Probabilidade de aleitamento materno exclusivo em menores de quatro meses, segundo idade em 1998 e 2000, no município do Rio de Janeiro* $(n=2242)$.

Table 1 - Likelihood of exclusive breastfeeding in infants under four months, according to age in 1998 and 2000, in the city of Rio de Janeiro* $(n=2,242)$.

\begin{tabular}{lcc}
\hline Idade (em dias) & AME (\%) & IC (95\%) \\
\hline Primeiro dia de vida & 39,6 & $0,35-0,44$ \\
30 & 32,1 & $0,29-0,35$ \\
60 & 23,3 & $0,22-0,25$ \\
90 & 16,0 & $0,14-0,18$ \\
120 & 12,4 & $0,10-0,15$ \\
\hline
\end{tabular}

*O modelo de regressão logística inclui as seguintes variáveis: idade da criança, idade e escolaridade da mãe, trabalho materno fora do lar, que mostraram associação no modelo de regressão logística apresentado na Tabela 5.

* The logistic regression model includes the following variables: child age, mother's age and schooling, mother's work outside home that showed association in the logistic regression model presented in Table 5. 
Tabela 2 - Distribuição de crianças menores de quatro meses de idade segundo características maternas e relacionadas ao nascimento. Município do Rio de Janeiro, 1998 e 2000.

Table 2 - Distribution of children under four months of age according to characteristics of mothers and related to birth. City of Rio de Janeiro, 1998 and 2000.

\begin{tabular}{lcc}
\hline & Variáveis & $\%$ \\
\hline Idade materna (anos) & $<=19$ & 21,0 \\
$(\mathrm{n}=2457)$ & 20 a 24 & 28,3 \\
& 25 a 29 & 22,8 \\
& 30 a 34 & 17,3 \\
& $>35$ & 10,5 \\
Escolaridade da materna & $1^{\circ}$ grau incompleto & 45,0 \\
(n=2358) & $1^{\circ}$ grau completo & 26,4 \\
& $2^{\circ}$ grau completo & 22,8 \\
& $3^{\circ}$ grau completo & 5,9 \\
Trabalho materno fora & Sim & 7,6 \\
do domicílio & Não & 92,4 \\
(n=2462) & & \\
Primiparidade & Sim & 45,8 \\
(n=2460) & Não & 54,2 \\
Peso ao nascer* & $<2500$ & 7,2 \\
(n=1265) & $2500 \mid-3000$ & 25,5 \\
& $>=3000$ & 67,4 \\
IHAC** & Sim & 11,1 \\
(n=2474) & Não & 88,9 \\
\hline
\end{tabular}

* Variável coletada somente no segundo ano de estudo.

* Variable collected only in the second year of study

** Iniciativa Hospital Amigo da Criança implantada no hospital onde a criança nasceu.

** Hospital Amigo da Criança Initiative implemented at the hospital where the child was born

materna. Da mesma forma, a escolaridade materna mostrou-se associada com o AME entre os menores de quatro meses (current status). Logo, quando se incluiu no modelo de regressão a variável AME no primeiro dia em casa, a escolaridade deixou de ter significância estatística na associação com o AME, o que corroborou a suspeita de que a primeira variável é um mecanismo intermediário na determinação da escolaridade materna sobre o AME em menores de quatro meses. Desta forma, optou-se por um modelo em que foi excluído o AME no primeiro dia após alta da maternidade.

Na análise multivariada, observou-se maior chance de amamentar exclusivamente entre as mães de maior escolaridade e as mães que não trabalhavam fora do domicílio.

\section{Discussão}

A estratégia de condução dos inquéritos sobre práticas alimentares no primeiro ano de vida durante as Campanhas Nacionais de Vacinação em local com alta cobertura desta intervenção tem se mostrado vantajosa em relação aos inquéritos tradicionais. Isto porque permite representatividade populacional, a um baixo custo e de forma rápida, sem interferir nas atividades de vacinação, além de favorecer a sensibilização dos profissionais envolvidos. No município do Rio de Janeiro, a cobertura das Campanhas Nacionais de Vacinação nos dois anos estudados foi de $98,3 \%$ e $97,8 \%$ nas primeira e segunda etapas de vacinação em 1998, e mais de $100 \%$ nas duas etapas em 2000.

O Instituto de Saúde e o Núcleo de 
Tabela 3 - Aleitamento materno exclusivo (AME) em crianças menores de quatro meses, segundo primiparidade, peso ao nascer, faixa etária, escolaridade e trabalho maternos e credenciamento ao IHAC. Município do Rio de Janeiro, 1998 e 2000.

Table 3 - Exclusive Breastfeeding (EBF) in children under four months, according to first parity, birth weight, age group, mother's schooling and work and credentialing at IHAC. City of Rio de Janeiro, 1998 and 2000.

\begin{tabular}{|c|c|c|c|}
\hline & Variáveis & $\%$ & Valor $\mathrm{p}$ \\
\hline \multirow{6}{*}{$\begin{array}{l}\text { Faixa etária materna } \\
(n=2445)\end{array}$} & $>19$ & 20,4 & \\
\hline & 20 a 24 & 20,5 & \\
\hline & 25 a 29 & 24,2 & \\
\hline & 30 a 34 & 25,4 & \\
\hline & $>35$ & 25,9 & \\
\hline & & & 0,122 \\
\hline \multirow{5}{*}{$\begin{array}{l}\text { Escolaridade da materna } \\
(\mathrm{n}=2347)\end{array}$} & $1^{\circ}$ grau incompleto & 20,1 & \\
\hline & $1^{\circ}$ grau completo & 24,1 & \\
\hline & $2^{\circ}$ grau completo & 24,3 & \\
\hline & $3^{\circ}$ grau completo & 34,1 & \\
\hline & & & 0,001 \\
\hline \multirow{3}{*}{$\begin{array}{l}\text { Trabalho materno fora do lar } \\
(n=2449)\end{array}$} & Sim & 11,2 & \\
\hline & Não & 23,7 & \\
\hline & & & 0,000 \\
\hline \multirow{3}{*}{$\begin{array}{l}\text { Primiparidade } \\
(\mathrm{n}=2447)\end{array}$} & Sim & 22,0 & \\
\hline & Não & 23,2 & \\
\hline & & & 0,477 \\
\hline \multirow{4}{*}{$\begin{array}{l}\text { Peso ao nascer* } \\
(n=1262)\end{array}$} & $<2500$ & 26,4 & \\
\hline & $2500 \mid-3000$ & 19,6 & \\
\hline & $>=3000$ & 25,0 & \\
\hline & & & 0,125 \\
\hline \multirow{3}{*}{$\begin{array}{l}\text { IHAC } \\
(n=2459)\end{array}$} & Sim & 26,4 & \\
\hline & Não & 22,2 & \\
\hline & & & 0,119 \\
\hline
\end{tabular}

* Variável coletada somente no segundo ano de estudo.

*Variable collected only in the second year of study

Pesquisas Epidemiológicas em Nutrição e Saúde da USP (Nupens), desde 1995, têm disseminado esta estratégia, assessorando alguns municípios brasileiros na realização de inquéritos epidemiológicos ${ }^{29}$.

Os inquéritos possibilitam estabelecer tendências das práticas alimentares na faixa etária estudada, assim como conhecer fatores determinantes destas práticas ${ }^{30}$.

A utilização de dados atuais sobre a alimentação (current status) apresenta-se como vantagem em relação à acurácia da informação uma vez que evita viés de memória na informação sobre alimentação das crianças ${ }^{19}$.

A informação sobre alimentação no primeiro dia em casa é, no entanto, retrospectiva, o que poderia constituir-se na principal limitação do estudo. Esse, contudo, é um dia marcante na vida da família, o que poderia beneficiar a lembrança dos fatos relativos ao cuidado e à alimentação da criança.

A medida de AME durante os quatro primeiros meses de vida, apesar de a recomen- 
Tabela 4 - Aleitamento materno exclusivo (AME) em menores de quatro meses*, segundo categorias de aleitamento materno no primeiro dia em casa após alta da maternidade, em 1998 e 2000, no município do Rio de Janeiro.

Table 4 - Exclusive Breastfeeding (EBF) in children under four months*, according to breastfeeding categories on the first day home after discharge from maternity in 1998 and 2000, in the city of Rio de Janeiro.

\begin{tabular}{lccc}
\hline Amamentação no $1^{\circ}$ dia em casa & AME $(\%)$ & Valor $p$ \\
\hline AM & $\operatorname{Sim}(n=2090)$ & 23,6 & 0,113 \\
& Não $(n=84)$ & 15,8 & \\
AME & $\operatorname{Sim}(n=1681)$ & 29,5 & 0,000 \\
& Não $(n=464)$ & 10,8 & \\
AMP & $\operatorname{Sim}(n=415)$ & 10,0 & 0,000 \\
& Não $(n=1710)$ & 26,4 & \\
\hline
\end{tabular}

* Análise restrita às crianças que tiveram alta da maternidade com até quatro dias após o nascimento.

*Analysis restricted to children discharged from the maternity up to four days after birth.

Tabela 5 - Aleitamento materno exclusivo (AME) em menores de quatro meses, segundo características maternas e do nascimento em 1998 e 2000, no município do Rio de Janeiro. (n $=2242$ ).

Table 5 - Exclusive Breastfeeding (EBF) in children under four months, according to characteristics of mothers and birth in 1998 and 2000, in the city of Rio de Janeiro. $(n=2,242)$

\begin{tabular}{llccc}
\hline Variáveis & & OR & IC (95\%) & Valor p \\
\hline Idade da criança & & 0,99 & $0,98-0,99$ & 0,000 \\
Idade materna & & 1,02 & $1,00-1,03$ & 0,018 \\
Escolaridade Materna & $1^{\circ}$ grau incompleto & 1,00 & - & - \\
& $1^{\circ}$ grau completo & 1,26 & $0,98-1,61$ & 0,067 \\
& $2^{\circ}$ grau completo & 1,24 & $0,96-1,62$ & 0,105 \\
& $3^{\circ}$ grau completo & 1,93 & $1,28-2,92$ & 0,002 \\
Trabalho materno & Não & 1,00 & - & - \\
Fora do domicílio & Sim & 0,59 & $0,36-0,96$ & 0,033 \\
IHAC & Não & 1,00 & - & - \\
& Sim & 1,22 & $0,89-1,67$ & 0,212 \\
\hline
\end{tabular}

dação ser até os seis meses, ainda é utilizada na maioria das publicações em virtude desta prática entre quatro e seis meses ainda ser pouco freqüente ${ }^{20,31}$.

Os resultados indicam a associação do AME entre os menores de quatro meses de idade, com escolaridade $\left(3^{\circ}\right.$ grau completo) e trabalho maternos e alimentação da criança nos primeiros dias de vida, ao passo que a idade materna, primiparidade, peso ao nascer e ter nascido em hospital amigo da criança não mostraram diferenças signi- ficativas nas freqüências de AME.

Algumas características da mulher estão associadas ao maior risco de introdução precoce de outros alimentos. No estudo nas capitais brasileiras, na região Sudeste, as mães que não trabalhavam fora e tinham ensino superior tiveram chance 2,73 vezes maior de amamentar exclusivamente do que as que trabalhavam fora e eram analfabetas $^{11}$. No presente estudo, ressalta-se que, muito embora a freqüência de AME tenha sido o dobro entre as mulheres que não 
trabalhavam fora do domicílio, apenas uma entre quatro mulheres que não trabalhavam fora de casa amamentava exclusivamente. Apesar de ser um fator facilitador, não estar afastada de casa devido ao trabalho não é condição suficiente para a garantia do AME.

A não associação da idade materna com a prática do AME não é consonante com achados de alguns estudos que indicam maiores chances de AME e de AM para mulheres mais velhas ${ }^{14}$.

A maior vulnerabilidade das mulheres de menor escolaridade pode estar relacionada ao menor acesso destes grupos a uma rede de suporte familiar/social, bem como a outros fatores facilitadores para a manutenção desta prática, como, por exemplo, acesso a serviços de atenção à saúde e à inserção formal no mercado de trabalho, usufruindo de benefícios legais como a licença maternidade ${ }^{32}$.

A alimentação da criança no primeiro dia em casa teve forte associação com o AME entre os menores de quatro meses, o que ratifica a necessidade das intervenções de promoção do AME nos primeiros dias de vida da criança ${ }^{33}$, que são essenciais para o sucesso da amamentação.

A Iniciativa Hospital Amigo da Criança (IHAC), que consiste na adequação de rotinas nas maternidades visando favorecer a amamentação, tem sido uma das principais estratégias utilizadas para a promoção desta prática, em face da reconhecida contribuição para o desmame precoce de práticas inadequadas nas maternidades. É evidente o impacto da IHAC na melhoria dos indicadores de amamentação, como demonstrado por alguns estudos ${ }^{34-36}$. O fato de não ter havido diferença significativa na chance de serem amamentadas exclusivamente entre as crianças que nasceram em maternidades que têm ou não o título de Hospital Amigo da Criança reflete, também, a complexidade de fatores ligados à decisão de amamentar, de fazê-lo de forma exclusiva e de mantê-lo durante um determinado intervalo de tempo ${ }^{32}$.

Por outro lado, apesar da importância das condutas visando à proteção do aleitamento materno nas maternidades, as ações visando à melhoria das práticas de aleitamento materno devem se dar em outros momentos da atenção à saúde da mulher e da criança ${ }^{32,33}$.

O acolhimento da mãe e do bebê e o suporte para o manejo adequado da amamentação no início da vida da criança, oferecendo escuta cuidadosa e orientação às dúvidas comuns neste momento, têm um importante papel no sucesso desta prática. $\mathrm{O}$ mesmo pode-se dizer das ações de promoção do aleitamento materno na atenção pré-natal ${ }^{33}$.

As abordagens acerca do aleitamento materno durante o atendimento pré-natal são decisivas para a garantia do exercício do direito da mulher de amamentar o seu filho, possibilitando reflexão sobre esta prática, conhecimento dos seus direitos e a preparação para o seu manejo. Da mesma forma, a atenção à mulher e à criança no puerpério deve ser capaz de intervir precocemente, acolhendo e dando escuta à mulher sobre as dificuldades do início desta prática, suas expectativas e desejos, não só em relação à amamentação, mas a outros aspectos de sua vida, garantindo a integralidade da atenção que é pressuposto básico dos programas de atenção à saúde da mulher e da criança. Esta abordagem em grande parte dos casos já é suficiente para auxiliar a mulher a superar os obstáculos deste momento, devendo ser acompanhada de orientação adequada sobre o manejo da lactação, segundo a especificidade de cada caso.

Em consonância com estas discussões e a partir de experiências de diversos países com estratégias de promoção ao aleitamento materno voltadas à rede básica de atenção à saúde, a exemplo da IHAC, mais recentemente, tem sido proposta a iniciativa unidade básica amiga da amamentação (IUBAAM). Esta proposta constitui importante aliada das estratégias já existentes ${ }^{37}$.

\section{Considerações finais}

Muito embora o setor saúde tenha papel 
importante na consolidação da "cultura da amamentação”, uma rede mais ampla de fatores interfere na prática do AME. Assim, colocado o papel do setor saúde e suas possibilidades de atuação para o pleno entendimento desta questão, deve-se retornar à discussão sobre a complexa determinação da amamentação, a qual ultrapassa os limites de intervenção desse setor. $\mathrm{O}$ ato de amamentar está condicionado por diversas questões, tendo forte determinação sociocultural e histórica, o que se pode observar com comparações dos padrões do aleitamento materno entre diferentes populações e através dos tempos ${ }^{15}$.

O suporte da comunidade e da família à mulher que amamenta é essencial para o sucesso da amamentação. Para tal, o entendimento dos fatores associados ao aleitamento materno e a identificação dos grupos de maior vulnerabilidade para esta prática são importantes para maior efetividade das ações e para a qualificação do atendimento prestado à mulher e à criança.

Dada a multiplicidade de questões que interferem na prática da amamentação, o monitoramento local de sua dinâmica e dos fatores a ela associados deve integrar as estratégias de sua promoção. Qualquer intervenção sobre a coletividade pressupõe que o seu planejamento seja pautado num diagnóstico real e atualizado, considerando as características da população local e os outros cenários existentes, que possam ter relação com o evento ou com a intervenção em si. Este diagnóstico deve ser contínuo, permitindo a identificação de tendências temporais e a avaliação das intervenções, uma vez que o processo de planejamento requer constante retro-alimentação.

\section{Referências}

1. World Health Organization. The optimal duration of exclusive breastfeeding - Results of a WHO systematic review. Geneve; 2001. Note for press no 7 . Acesso por <http:// www.who.int> em outubro de 2001.

2. Ferreira L, Parada CMGL, Carvalhaes, MABL. Tendência do aleitamento materno em município da região centrosul do estado de São Paulo: 1995-1999-2004. Rev Nutr 2007; 20 (3): 265-73.

3. Castro IRR, Damião JJ, Monteiro CA, Gomes MASM, Azevedo AMF, Brasil CLP, Tavares EL, Engstron EM. Breast-feeding Surveillance System (BSS) nested in national days of vaccination: design and findings in the city of Rio de Janeiro (Rio) - Brazil. In: Abstracts of International Congress of Nutrition; 2001. Vienna: Austrian Nutrition Society; 2001. p. 437.

4. Passos MC, Lamounier JA, Silva CAM, Freitas SNF, Baudson MFR. Práticas de amamentação no município de Ouro Preto, MG, Brasil. Rev Saúde Pública 2000; 34 : 617-22.

5. Brasil CLP, Tavares EL, Castro, IRR, Gomes MAM. Situação do aleitamento materno no município do Rio de Janeiro / 1996 e 1998. Saúde em Foco 1999; 8: 22-6.

6. Venâncio SI, Monteiro CA. A tendência da prática da amamentação no Brasil nas décadas de 70 e 80. Rev Bras Epidemiol 1998; 1: 40-9.

7. Monteiro CA. O panorama da nutrição infantil nos anos 90. Cadernos de Políticas Sociais (Série documentos para discussão ${ }^{\circ}$ 1). São Paulo: UNICEF; 1997.
8. Horta BL, Olinto MTA, Victora CG, Guimarães PRV. Amamentação e padrões alimentares em crianças de duas coortes de base populacional no Sul do Brasil: tendências e diferenciais. Cad Saúde Pública 1996; 12(S): 43-8.

9. PNIAM/INAN/UNICEF. Aleitamento Materno e o Município. Rio de Janeiro: Editora Coronário; 1995.

10. Rea MF. Reflexões sobre a amamentação no Brasil: de como passamos a 10 meses de duração. Cad Saúde Pública 2003; 19(S1): S37-45.

11. Ministério da Saúde. Prevalência do Aleitamento Materno nas Capitais Brasileiras e no Distrito Federal. Brasília: MS; 2001 (Relatório de pesquisa).

12. Kummer SC, Giugliani ERJ, Susin LO, Folletto JL, Lermen NR, Wu VYJ, Santos L, Caetano MB. Evolução do padrão de aleitamento materno. Rev Saúde Pública 2000; 34: 143-8.

13. Weiderpass E, Barros F C, Victora CG, Tomasi E, Halpern R. Incidência e duração da amamentação conforme o tipo de parto: estudo longitudinal no Sul do Brasil. Rev Saúde Pública 1998; 32: 225-31.

14. Gigante D P, Victora CG, Barros FC. Nutrição materna e duração da amamentação em uma coorte de nascimento de Pelotas, RS. Rev Saúde Pública 2000; 34: 259-65.

15. Almeida JAG. Amamentação: Um Híbrido Naturezacultura. Rio de Janeiro: Editora Fiocruz; 1999. 
16. Horta BL, Victora CG, Gigante DP et al. Duração da amamentação em duas gerações. Rev Saúde Pública 2007; 41(1): 13-8.

17. Franca GVA, Brunken GS, Silva SM et al. Determinantes da amamentação no primeiro ano de vida em Cuiabá, Mato Grosso. Rev Saúde Pública 2007; 41(5): 711-8.

18. Oliveira LPM, Assis AMO, Gomes GSS, Prado M, Barreto, ML. Duração do aleitamento materno, regime alimentar e fatores associados segundo condições de vida em Salvador, Bahia, Brasil. Cad Saúde Pública 2005; 21(5): 1519-30.

19. Venâncio SI, Escuder MML, Kitoko P, Rea MF, Monteiro CA. Freqüência e determinantes do aleitamento materno em municípios do Estado de São Paulo. Rev Saúde Pública 2002; 36(3): 313-8.

20. Sena MCF, Silva EF, Pereira MG. Prevalência do aleitamento materno nas capitais brasileiras. Rev Assoc Med Bras 2007; 53(6): 520-524.

21. Brasil CLP, Tavares EL, Castro IRR, Gomes MAM. Situação do aleitamento materno no município do Rio de Janeiro / 1996 e 1998. Saúde em Foco 1999; 8: 22-6.

22. Castro IRR, Silva MAV, Cardoso LO, Damião JJ, Rito RVVF, Engstrom EM. Evolução do Aleitamento Materno no Município do Rio de Janeiro no período de 1996 a 2003. Publicado em Rio Estudos, no 136, Set/2004. Coleção Estudos da Cidade, Outubro de 2004. http:// www.armazemdedados.rio.rj.gov.br. Acessado em 5 de fevereiro de 2007.

23. Cochran WG. Sampling Techniques. $3^{\text {a. }}$ Ed. New York: Wiley; 1977.

24. Organizacion Panamericana de la Salud / Organizacion Mundial de la Salud. Indicadores para evaluar las practicas de lactancia materna. Genebra: OMS/CED/ SER; 1991.

25. Kleinbaum DG. Logistic Regression - A Self-Learning Text. New York: Spring Verlag; 1994.

26. Rothaman KJ, Greenland S. Modern Epidemiology. United States of America: Lippincott-Raven Publishers HL; 1998.
27. Dean AG, Dean JA, Coulombier D, Brendel KA, Smith DC, Burton AH et al. Epi Info, version 6: a word processing database and statistics program for epidemiology on microcomputers. Atlanta: Centers of Disease Control and Prevention; 1994.

28. StataCorp. Stata Statistical Software: Release 8. College Station, Texas: Stata Corporation; 2003.

29. Venâncio SI, Escuder MML, Kitoko P et al. Freqüência e determinantes do aleitamento materno em municípios do Estado de São Paulo. Rev Saúde Pública 2002; 36(3): 313-8.

30. Saldiva SRDM, Escuder MM, Mondini L et al. Práticas alimentares de crianças de 6 a 12 meses e fatores maternos associados. J Pediatr 2007; 83 (1): 53-8.

31. Silva AP, Souza N. Prevalência do aleitamento materno Rev Nutr 2005; 18(3): 301-10.

32. Faleiros FTV, Trezza EMC, Carandina L. Aleitamento materno: fatores de influência na sua decisão e duração. Rev Nutr 2006; 19(5): 623-30.

33. Oliveira MIC, Gomes MAM. As Unidades Básicas Amigas da Amamentação: uma nova tática no apoio ao aleitamento materno. In: Rego JD. Aleitamento Materno. São Paulo: Editora Atheneu; 2001. p. 343-6.

34. Cattaneo A, Buzzetti R.. Effect on rates on breast-feeding of training for Baby Friendly Hospital Initiative. BJM 2001; 323(8): 1358-62.

35. Braun ML, Giugliani E.J. Avaliação do impacto da Iniciativa Hospital Amigo da Criança nas taxas de aleitamento materno. In: Resumos do III Congresso Brasileiro de Bancos de leite Humano, 2002. Petrópolis: Fundação Oswaldo Cruz/Ministério da Saúde; 2002. p. 136.

36. Lamounier JA. A experiência iniciativa hospital amigo da criança. Rev Assoc Méd Bras 1998, 44(4): 319-24.

37. Oliveira MIC, Camacho LAB, Souza IEO. Promoção, proteção e apoio à amamentação na atenção primária à saúde no Estado do Rio de Janeiro, Brasil: uma política de saúde pública baseada em evidência. Cad Saúde Pública 2005; 21(16): 1901-10.

Recebido em: 17/10/07

Versão final reapresentada em: 04/06/08 Aprovado em: 25/06/08 